\title{
Parasiticidal effect of synthetic bovine Lactoferrin peptides on the enteric parasite Giardia intestinalis
}

\begin{tabular}{|c|c|}
\hline Journal: & Biochemistry and Cell Biology \\
\hline Manuscript ID & bcb-2016-0079.R5 \\
\hline Manuscript Type: & Article \\
\hline Date Submitted by the Author: & $16-N o v-2016$ \\
\hline Complete List of Authors: & $\begin{array}{l}\text { Aguilar-Diaz, Hugo; Facultad de Medicina, Universidad Autónoma de } \\
\text { Sinaloa, CIASaP } \\
\text { Canizalez-Roman, Adrian; Facultad de Medicina, Universidad Autonoma de } \\
\text { Sinaloa, CIASaP; Hospital de la Mujer, Departamento de Investigación } \\
\text { Nepomuceno-Mejia, Tomas; Instituto Nacional de Salud Pública., Centro } \\
\text { Regional de Investigación en Salud Pública } \\
\text { Gallardo-Vera, Francisco; Facultad de Medicina. Universidad Nacional } \\
\text { Autónoma de México, Departamento de Biologia Celular y Tisular } \\
\text { Hornelas-Orozco, Yolanda; Universidad Nacional Autónoma de México } \\
\text { Nazmi, Kamran; Academic Center Dentistry Amsterdam, University of } \\
\text { Amsterdam and VU University Amsterdam, Department of Oral } \\
\text { Biochemistry } \\
\text { Bolscher, Jan; Academic Center Dentistry Amsterdam, University of } \\
\text { Amsterdam and VU University Amsterdam, Department of Oral } \\
\text { Biochemistry } \\
\text { Carrero, Julio Cesar; Universidad Nacional Autonoma de Mexico Instituto } \\
\text { de Investigaciones Biomedicas } \\
\text { Leon-Sicairos, Claudia; Universidad Autónoma de Sinaloa, Programa } \\
\text { Regional de Noroeste para el Doctorado en Biotecnología. FCQB } \\
\text { Leon-Sicairos, Nidia; Facultad de Medicina, Universidad Autónoma de } \\
\text { Sinaloa, CIASaP; Hospital Pediátrico de Sinaloa, Departamento de } \\
\text { investigación }\end{array}$ \\
\hline Keyword: & Lactoferrin, Peptides, LFchimera, parasiticidal, Giardiasis \\
\hline
\end{tabular}

\section{SCHOLARONE ${ }^{m}$ \\ Manuscripts}




\section{Parasiticidal effect of synthetic bovine Lactoferrin peptides on}

\section{2 the enteric parasite Giardia intestinalis}

3

Hugo Aguilar-Diaz ${ }^{1}$, Adrian Canizalez-Roman ${ }^{1,2}$, Tomas Nepomuceno-Mejia ${ }^{3}$, Francisco Gallardo-Vera ${ }^{4}$, Yolanda Hornelas-Orozco ${ }^{5}$, Kamran Nazmi ${ }^{6}$, Jan G.M. Bolscher ${ }^{6}$, Julio Cesar Carrero $^{7}$, Claudia Leon-Sicairos ${ }^{8}$, Nidia Leon-Sicairos ${ }^{1,9^{*}}$

1 CIASaP, Facultad de Medicina, Universidad Autónoma de Sinaloa. Cedros y Sauces, Fracc. Fresnos Culiacán 80246, Sinaloa, México

2 Departamento de Investigación, Hospital de la Mujer. Boulevard Miguel Tamayo Espinoza de los Monteros, S/N Col. Desarrollo Urbano Tres Ríos. Culiacán 80020, Sinaloa, México

${ }^{3}$ Centro Regional de Investigación en Salud Pública, Instituto Nacional de Salud Pública. Calle 4a. Avenida Norte esquina con Calle 19 Pte S/N, Centro, Tapachula 30700, Chiapas, Mexico

${ }^{4}$ Laboratorio Inmunobiología, Departamento de Biología Celular y Tisular, Facultad de Medicina. Universidad Nacional Autónoma de México. Ciudad Universitaria, México DF 04510, México

${ }^{5}$ Servicio Académico de Microscopía Electrónica de Barrido. Instituto de Ciencias del Mar y Limnología, Universidad Nacional Autónoma de México, México, D. F. 04510, México

${ }^{6}$ Department of Oral Biochemistry, Academic Centre for Dentistry Amsterdam, University of Amsterdam and VU University, 1081 LA, Amsterdam, The Netherlands

${ }^{7}$ Departamento de Inmunología, Instituto de Investigaciones Biomédicas, Universidad Nacional Autónoma de México. Ciudad Universitaria, México, DF 04510, México

${ }^{8}$ Facultad de Ciencias Químico-Biológicas, Universidad Autónoma de Sinaloa, Avenida de las Américas y Josefa Ortiz (Ciudad Universitaria), Culiacán 80030, Sinaloa, México

${ }^{9}$ Departamento de Investigación, Hospital Pediátrico de Sinaloa. Blvd. Constitución S/N, col. Jorge Almada, Culiacan 80200, Sinaloa, México

Corresponding author. Nidia León-Sicairos. Email nidialeon@uas.edu.mx

CIASaP, Facultad de Medicina, Universidad Autónoma de Sinaloa, Cedros y Sauces S/N

Fracc. Fresnos. Culiacán Sinaoa, 80246 México Telephone +52 6672278588 Fax +52 66 
41 Giardia intestinalis is the most common infectious protozoan parasite in children. Despite

42 the effectiveness of some drugs, the disease remains a major worldwide problem.

43 Consequently, the search for new treatments is important for disease eradication. Biological

\section{Abstract} molecules with antimicrobial properties represent a promising alternative to combat pathogens. Bovine lactoferrin (bLF) is a key component of the innate host defense system, and its peptides have exhibited strong antimicrobial activity. Based on these properties, we evaluated the parasiticidal activity of these peptides on G. intestinalis. Trophozoites were incubated with different peptide concentrations for different periods of time, and the growth or viability was determined by carboxyfluorescein-succinimidyl-diacetate-ester (CFDA) and propidium iodide (PI) staining. Endocytosis of peptides was investigated by confocal microscopy, damage was analyzed by transmission and scanning electron microscopy, and the type of programmed cell death was analyzed by flow cytometry. Our results showed that the LFpeptides had giardicidal activity. The LFpeptides interacted with G. intestinalis and exposure to LFpeptides correlated with an increase in the granularity and vacuolization of the cytoplasm. Additionally, the formation of pores, extensive membrane disruption, and programmed cell death was observed in trophozoites treated with LFpeptides. Our results demonstrate that LFpeptides exhibit potent in vitro antigiardial activity.

\section{Keywors: Lactoferrin; LFchimera; parasiticidal; peptides; Giardia; Giardiasis}


64

65

66

67

68

69

81 Giardia species have two major stages in their lifecycle. Infection with G. intestinalis

82 initiates when the cysts are ingested with contaminated water or, less commonly, food or

83 through direct fecal-oral contact. The cyst is relatively inert, allowing prolonged survival in 84 a variety of environmental conditions (Adam 2001; Carranza and Lujan 2010). After 85 exposure to the acidic environment of the stomach, cysts excyst into trophozoites in the 86

\section{Introduction}

Giardia intestinalis (also known as Giardia lamblia or Giardia duodenalis), is a flagellated unicellular eukaryotic parasite that causes giardiasis, a diarrheal disease, throughout the world (Watkins and Eckmann 2014). Giardiasis is the most common cause of waterborne outbreaks of diarrhea in the United States and is occasionally considered a cause of foodborne diarrhea (Furness et al. 2000). In certain areas of the world, water contaminated with G. lamblia commonly causes travel-related giardiasis in tourists (Painter et al. 2015; Watkins and Eckmann 2014). This parasite is particularly problematic in developing countries, where a very high prevalence and incidence of infection has been reported. Data suggest that long-term growth retardation in children can result from chronic giardiasis, in part due to the parasite attaching itself to the lining of the small intestine in humans, where it interferes with the body's absorption of fats and carbohydrates from digested foods (Eckmann 2003). Giardiasis is reported more frequently in young children and immunocompromised or chronically ill individuals, and $G$. intestinalis infection is particularly significant for people with malnutrition, immunodeficiencies, or cystic fibrosis (Painter et al. 2015; Watkins and Eckmann 2014). proximal small intestine. The trophozoite (the vegetative form) replicates in the small 
87 intestine, causing symptoms of diarrhea and malabsorption. After exposure to biliary fluid,

88 some of the trophozoites form cysts in the jejunum and are passed in the feces, allowing for 89 completion of the transmission cycle by infecting a new host (Adam 2001; Carranza and 90 Lujan 2010).

91

92 Standard treatment for giardiasis consists of antibiotic therapy. Metronidazole is the most 93 commonly prescribed drug for this condition. However, metronidazole use has been 94 associated with significant failure rates in clearing parasites from the gut and with poor 95 patient compliance (Watkins and Eckmann 2014). In addition, an increasing incidence of 96 nitroimidazole-refractory giardiasis has been reported in travelers from India (Nabarro et al.

97 2015). Appropriate fluid and electrolyte management is critical, particularly in patients with 98 large-volume diarrheal losses and in children with acute or chronic diarrhea who manifest a 99 failure to thrive, malabsorption, or other gastrointestinal tract symptoms in whom Giardia 100 organisms have been identified (Dominguez-Lopez et al. 2011; Hill 1993; Vesy and 101 Peterson 1999). Innate-immunity mechanisms play a role in the control and/or severity of the infection; 104 however, little is known about the mechanisms involved in this immune response 105 (Roxstrom-Lindquist et al. 2006). Breastfeeding protects infants from $G$. intestinalis 106 infection. Breast milk contains detectable titres of secretory $\operatorname{IgA}$, which is protective for 107 infants, especially in developing countries (Eckmann 2003; Morrow et al. 1992). A study 108 from Egypt showed breast-fed infants had a lower incidence of symptomatic and 109 asymptomatic infection. Furthermore, infected infants who were exclusively breast-fed had 110 fewer clinical manifestations than those who were not exclusively breast-fed (Abdel-Hafeez 
111 et al. 2013; Gendrel et al. 1989). Apart from IgA, a milk protein called lactoferrin (LF) has

112 been reported as an immunological factor that kills trophozoites in vitro and in vivo: both

$113 \mathrm{hLf}$ and bLF and peptides derived from their N-terminals (LfcinH and LfcinB) have

114 microbicidal activity against Giardia (Gillin et al. 1983; Ochoa et al. 2008; Turchany et al.

115 1995; Turchany et al. 1997). However, it is not known if synthetic bLF peptides share this

116 activity and the mechanism of action of Lf-derived peptides is unknown. Thus, the aim of

117 this work was to study the possible microbicidal activity of synthetic bLF-based peptides

118 against Giardia intestinalis and to explore the mechanism involved in parasitical effects

119 against Giardia in vitro.

120

121

122

123

124

125

126

127

128

129

130

131

132

133

134 
135

136

137

138

139

140

141

142

143

144

145

146

147

148

149

150

151

152

153

154

155

156

157

\section{Materials and methods}

\section{Bovine lactoferrin, synthetic peptides and chemotherapeutic agents}

Bovine LF (bLF, 20\% iron saturated) was kindly donated by Morinaga Milk Industries Co

(Tokyo, Japan). The purity of bLF (>98\%) was checked by SDS-PAGE stained with silver nitrate. Lactoferrin concentration was assessed by UV spectroscopy on the basis of an extinction coefficient of $15.1(280 \mathrm{~nm}, 1 \%$ solution). The bLF iron saturation was about $20 \%$ as detected by optical spectroscopy at $468 \mathrm{~nm}$ on the basis of an extinction coefficient of $0.54(100 \%$ iron saturation). LPS contamination of bLf, estimated by Limulus Amebocyte assay (LAL Pyrochrome kit, ThermoFicherScientific, Waltham, MA, USA), was equal to $0.7 \pm 0.06 \mathrm{ng} / \mathrm{mg}$ of bLF. Synthetic peptides LFcin17-30, LFampin265-284 and LFchimera were obtained by solid-phase peptide synthesis using Fmoc chemistry, as described previously (Bolscher et al. 2012; Bolscher et al. 2009). The chemotherapeutic agents used were metronidazole and albendazole (Sigma Chemical Co., St. Louis, MO, USA). Stock solutions were prepared in phosphate-buffered saline for metronidazole and dimethyl sulphoxide (DMSO) for albendazole. The final DMSO concentration in the culture tubes was $<0.5 \%(\mathrm{v} / \mathrm{v})$.

\section{Giardia intestinalis cyst isolation}

Human fecal samples from different patients containing abundant $G$. intestinalis cysts were obtained from children at Hospital Pediatrico de Sinaloa in Culiacan City. G. intestinalis cysts were purified and concentrated from feces using a combined sucrose flotation and simplified sucrose gradient method (Hautus et al. 1988). The cysts, after being washed twice in distilled water, were resuspended in distilled water and stored at $4^{\circ} \mathrm{C}$ for a 
158

159

160

161

162

163

164

165

166

167

168

169

170

171

172

173

174

175

176

177

178

179

180

181

maximum of 3 days prior to use. The G. intestinalis-positive cysts were confirmed by light microscopy and PCR (Elsafi et al. 2013; Stojecki et al. 2014).

\section{Excystation and axenization}

G. intestinalis cysts were purified and concentrated from feces by combining the sucrose flotation method with a simplified sucrose gradient method. The excystation procedure was a modification of the Bingham and Meyer technique performed by Schupp et al. (1988). Briefly, the isolation procedure involved three steps: the concentration and cleaning of cysts by centrifugation in sucrose gradients performed 1 to 3 days after collection, the induction of excystation performed in acid solution from 1 to 5 days after cleaning cyst suspensions, and the culture and axenization in modified TYI-S-33 medium (Schupp et al. 1988).

\section{Viability and growth inhibition assays}

The effect of bLF, LFpeptides and chemotherapeutic agents on the long-term viability or permabilization of $G$. intestinalis trophozoites was determined by the inclusion of carboxyfluorescein-succinimidyl-diacetate-ester (CFDA) (St. Louis, MO, USA), or the exclusion of the dye propidium iodide (PI).

In one set of experiments, cultures were initiated by the addition of $2.5 \times 10^{4}$ trophozoites in $0.1 \mathrm{ml}$ of medium to vials $(15 \mathrm{x} 45 \mathrm{~mm})$ containing $3.9 \mathrm{ml}$ of medium containing none (optimal viability) or one of the following agents: $1,5,10,20$, or $40 \mu \mathrm{M}$ of bLF, LFcin17-30, LFampin265-284, or LFchimera. As control of growth inhibition, treatments with metronidazole $(1,5,10,20$, and $40 \mu \mathrm{M})$ were used. The vials were incubated at $37^{\circ} \mathrm{C}$ for $12 \mathrm{~h}$, chilled on ice to detach trophozoites, and centrifuged at $500 \mathrm{~g}$ for $10 \mathrm{~min}$, and the pellet was resuspended in $1 \mathrm{ml}$ of medium. Long-term viability was determined using the fluorescent probe carboxyfluorescein-succinimidyl-diacetate-ester 
182 (CFDA-SE) $(10 \mu \mathrm{g} / \mathrm{ml})$ and visualized by epi-fluorescense microscopy (dos Santos et al. 183 2015). Experiments were performed at least three times in triplicate, and the mean and 184 standard deviations are indicated. Comparison of means was done by using a two-tailed t185 test for independent samples. A value of $\mathrm{P}<0.05$ was considered statically significant.

186 In other experiments, G. intestinalis trophozoites $\left(10^{6}\right)$ were placed in tubes with 187 TYI-S-33 and were then incubated alone (optimal viability) or with $100 \mu \mathrm{M}$ metronidazole, $1885 \mu \mathrm{M}$ albendazole, or $40 \mu \mathrm{M}$ LFcin17-30, LFampin265-284 or LFchimera for $2 \mathrm{~h}$ at $37^{\circ} \mathrm{C}$. 189 Membrane permeabilization by propidium iodide (PI) was used as a measure of trophozoite 190 viability. The total number of organisms per vial was counted and compared to that of 191 parallel untreated cultures. Experiments were performed at least three times in triplicate, 192 and the mean and standard deviations are indicated. Comparison of means was done by 193 using a two-tailed t-test for independent samples. A value of $\mathrm{P}<0.05$ was considered 194 statically significant.

195 Tubes with trophozoites were also incubated with combinations of metronidazole \pm 196 LFpeptides or albendazole \pm LFpeptides or LFpeptides alone. Membrane permeabilization 197 of propidium iodide (PI) measured by flow cytometry was used as a measure of trophozoite 198 viability.

200 Confocal microscopy

201 Trophozoites $\left(10^{6} / \mathrm{ml}\right.$ in TYI-S-33 medium) were incubated with $2 \mu$ M FITC-LFcin17-30, 202 FITC-LFampim265-284 or FITC-LFchimera for $0,5,15,30,45$ or $60 \mathrm{~min}$ at $37^{\circ} \mathrm{C}$. After 203 washing twice in cold PBS, trophozoites were collected by centrifugation, fixed with $4 \% p$ 204 formaldehyde $\left(30 \mathrm{~min}\right.$ at $\left.37^{\circ} \mathrm{C}\right)$, permeabilized with $0.5 \%$ triton $\mathrm{X}-100$, counterstained with 205 PI, washed and processed for analysis by confocal microscopy. 
206

207

208

209

210

211

212

213

214

215

216

217

218

219

220

221

222

223

224

225

226

227

228

229

\section{Transmission electron microscopy}

G. intestinalis trophozoites (approximately $10^{6}$ cells) were untreated or treated with $40 \mu \mathrm{M}$ of bLF-peptides for $2 \mathrm{~h}$ at $37^{\circ} \mathrm{C}$. Trophozoites were collected by centrifugation and processed for standard transmission electron microscopy (Vázquez-Nin and Echeverría 2000). Briefly, samples were fixed using 2.5\% glutaraldehyde in PBS for $2 \mathrm{~h}$ at $4^{\circ} \mathrm{C}$, postfixed in $1 \%$ osmium tetroxide for $1 \mathrm{~h}$, dehydrated in a graded series of ethanol, and embedded in Epon resin. Semi-thin sections (approximately 300-400 nm) were stained with toluidine blue and observed with bright-field microscopy. Sections were mounted on copper grids and contrasted with uranyl acetate and lead citrate. Samples embedded in the resin were cut with an ultramicrotome. Serial thin sections (50-60 nm width) were obtained, and the samples were then stained with uranyl acetate and lead citrate. The sections were observed with a JEOL 1010 transmission electron microscope at $80 \mathrm{kV}$.

\section{Scanning electron microscopy}

G. intestinalis trophozoites $\left(5 \times 10^{6}\right)$ were incubated with $40 \mu \mathrm{M}$ of LFpeptides for $2 \mathrm{~h}$ at $37^{\circ} \mathrm{C}$, washed and fixed with $10 \%$ formaldehyde for $72 \mathrm{~h}$. Samples were washed and then dehydrated in a graded series of ethanol. Dehydrated samples were placed in acetone and then collocated in desiccator for $30 \mathrm{~min}$. Finally samples were mounted in glass chambers and bombarded with gold particles for $20 \mathrm{~min}$ (Perez-Rangel et al. 2013). Processed specimens were observed with a scanning electron microscope JEOL LSM6360LV.

\section{Flow cytometry}

To detect early apoptosis or programmed cell death, G. intestinalis trophozoites $\left(5 \times 10^{6}\right)$ treated with $40 \mu \mathrm{M}$ LFpeptides for $2 \mathrm{~h}$ at $37^{\circ} \mathrm{C}$ were stained with Allophycocyanin-Annexin and 7-Amino-Actinomicyn D (BD Pharmingen, San José, CA), following the manufacturer's instructions. Annexin V (also known as Annexin A5) and 7-AAD (7-amino- 
230 actinomycin D) are indicators of early apoptosis or programmed cell death in Giardia and

231 necrosis or late programmed cell death, respectively. Both fluorescent dyes were measured 232 in G. intestinalis using Acoustic Focusing Cytometer AttuneTM Blue/Red (Life 233 Technologies).

235 Results

236 Effects of bLF, LFpeptides and chemotherapeutics agents on the viability and long237 term growth of Giardia intestinalis

238 The effect of LFcin17-30, LFampin265-284 and LFchimera on G. intestinalis trophozoites 239 was analyzed using the membrane permeabilization probe PI, as a measure of viability. 240 When the G. intestinalis trophozoites were incubated with $40 \mu \mathrm{M}$ LFpeptides for $2 \mathrm{~h}$, the 241 trophozoites showed a marked PI uptake, indicating a drastic effect on the viability (Figure

242 1, panel A). LFchimera had the best killing effect (more than $98 \%$ of the trophozoites were 243 stained with PI) followed by the other LFpeptides (70-80\% of the trophozoites were 244 stained) compared with untreated cells. Furthermore, the parasiticidal effect of LFchimera 245 was higher than the drugs albendazole and metronidazole (controls of viability inhibition) 246 (Figure 1, panel A).

247 Concentrations of $100 \mu \mathrm{M}$ of metronidazole or $5 \mu \mathrm{M}$ of albendazole were needed to 248 permeate more than $95 \%$ of $G$. intestinalis cultures. However, only $20 \mu \mathrm{M}$ and $3 \mu \mathrm{M}$ of 249 these drugs were needed to reach the same level of membrane permeation when 20, 30 or 5 $250 \mu \mathrm{M}$ of LFcin17-30, LFampin254-284 or LFchimera, respectively, were added, (Table 1). 251 Thus, the combined effect of lower concentrations of metronidazole or albendazole with 252 LFcin17-30, LFampin265-284 or LFchimera had the best initial parasiticidal activity (Table $2531)$. 
254 Next, the ability of different concentrations of LFcin17-30, LFampin265-284 and 255 LFchimera to inhibit long-term growth was tested using the live-stain CFDA. After $12 \mathrm{~h}$ of 256 interaction, the growth of $G$. intestinalis trophozoites in the presence of 5, 10, 20 or $40 \mu \mathrm{M}$ 257 of LF and LFpeptides was lower than that found in the untreated trophozoites (Figure 1, 258 panel B). Interestingly, LFchimera had better giardicidal activity than the drug 259 metronidazole: $40 \mu \mathrm{M}$ LFchimera inhibited the growth of cultures with more efficacy than $26040 \mu \mathrm{M}$ metronidazole. Similar results were obtained when $G$. intestinalis cultures were 261 incubated with $40 \mu \mathrm{M}$ of each treatment for longer periods of time $(24,36$ and $48 \mathrm{~h}$, data 262 not shown). Therefore, at $40 \mu \mathrm{M}$ LFchimera had the best parasiticidal activity, followed by $26340 \mu \mathrm{M}$ of the drug metronidazole and LFcin17-30, LFampin 265-284, and bLF.

\section{LFcin17-30, LFampin265-284 and LFchimera interact with Giardia intestinalis}

266 The majority of live trophozoites incubated with $2 \mu \mathrm{M}$ FITC-Labeled LFcin17-30, 267 LFampin265-284 or LFchimera showed bright green fluorescence (Figure 2, panels B, C 268 and D, respectively). Trophozoites were counterstained with PI, which exhibits red 269 fluorescence (Figure 2, panels A-D). At $30 \mathrm{~min}$, all FITC-LFpeptides were visible in the 270 trophozoites (green fluorescence, arrowheads), suggesting that the LFpeptides were 271 endocytosed or internalized by $G$. intestinalis. Additionally, it would appear that degraded 272 RNA and DNA are present in the images of trophozoites treated with the peptides (B-D). 273 The controls (untreated trophozoites) were negative for the green fluorescence (A).

275 Morphologic effects of LFcin17-30, LFampin265-284 and LFchimera on Giardia 276 intestinalis trophozoites 
277 We examined cells after relatively short times of exposure to observe early and more direct

278 changes, as well as those accompanying or secondary to cell lysis, by transmission electron 279 microscopy (TEM). Untreated cells had a smooth cellular membrane (cm, double-headed 280 arrow), peripheral vacuoles (pv, lines) near the cellular membrane, three pairs of flagella 281 (F, discontinuous arrow), adherent disk (ad), and two nuclei (N) and two nucleoli (no)

282 (Figure 3, panel A). Magnification of the picture shows granules of electron-dense material 283 (asterisk) distributed in the cytoplasm and the arrangement of the microtubules belonging to 284 the adherent disk (ad) (panel B). Exposure to LFcin17-30 led to profound intracellular 285 changes, such as an increase in electron-dense material in the cytoplasm (asterisk), 286 reorganization of the flagella ( $\mathrm{F}$, discontinuous arrow) and displacement of the adherent 287 disk (ad, arrow), Figure 3, panel C. In the magnified picture, there are no peripheral 288 vacuoles (pv) near the cellular membrane (cm, double-headed arrow), Panel D. Treatment 289 with LFampin265-284 also caused intracellular damage, including an increase in electron290 dense material (asterisk), reorganization of the flagella ( $\mathrm{F}$, discontinuous arrow), a large 291 hole in the cytoplasm (arrowhead), and also disruption in the cellular membrane (cm, 292 double-headed arrow) (Figure 3, panel E). The magnified picture shows the large hole 293 induced by treatment with LFampin265-284 (arrowhead, panel F). Trophozoites treated 294 with LFchimera produced the most significant changes and damage in the trophozoites. 295 There were marked changes in the electron-dense material in the cytoplasm (asterisks), the 296 flagella (F, discontinuous arrow) were disrupted and reorganized, and the cytoplasm 297 showed large holes (arrowheads) in which some electron-dense material is visible (Figure 298 3, panel G). In the magnified picture the larges holes (arrowheads) with aggregates inside 299 them (asterisks) can be seen in more detail (Panel H). Shrunken and distorted peripheral 300 vacuoles (pv, lines) were also observed. 
301

302

303

304

305

G. intestinalis trophozoites treated with LFpeptides also were analyzed under scanning electron microscopy (SEM). Treated trophozoites exhibited damage on the cell surface (Figure 4). By SEM it was found that G. intestinalis cultures treated with LFcin17-30 (B), LFampin265-284 (C), or LFchimera (D) showed alterations in size, irregular form and perforations (arrows) compared with untreated trophozoites (which had the typical structure of G. intestinalis trophozoites) (A). Additionally, the large hole in the membrane of the trophozoite treated with Lfchimera shown in panel D revealed the presence of unusual aggregates.

\section{LFcin17-30, LFampin265-284 and LFchimera induced programmed cell death in}

\section{Giardia intestinalis trophozoites}

LFcin17-30, LFampin265-284 and LFchimera induced early programmed cell death in $G$. intestinalis trophozoites treated with $40 \mu \mathrm{M}$ of these peptides for $2 \mathrm{~h}$ (Figure 5, panels A, B, and C, respectively). The quadrants represent Q1: Necrotic cells, Q2: Necrosis and apototic cells, Q3: Earlier apoptotic cells, Q4: live cells. Cells treated with LFchimera and LFampin265-284 induced early programmed cell dead as measured by the liberation of phosphatidylserine (PS) (Q3. 27.9 and 25.5\%, respectively), and, to a lesser extent, early programmed cell death was also induced by treatment with LFcin17-30 (Q3. 7.66\%). Although the total of the analyzed population were not undergoing programmed cell death, this result and the images observed under electron microscopy reinforce the idea that $G$. intestinalis is induced to undergo programmed cell death after treatment with LFpeptides. 
325

326

327

328

329

\section{Discussion}

Although giardiasis has been a threat to mankind for thousands of years, this parasitic infection has been, until recently, relatively neglected. G. intestinalis is a major cause of parasite-induced diarrhea in humans and animals and is currently an important public health problem, mostly in developing countries but also in developed countries (Escobedo et al. 2010; Painter et al. 2015). Nearly 33\% of people in developing countries have had giardiasis, and nearly $2 \%$ of adults and $6 \%$ to $8 \%$ of children have giardiasis worldwide (Escobedo et al. 2010; Furness et al. 2000; Painter et al. 2015). Although giardiasis is considered by most medical practitioners to be an easily treatable infection, prolonged symptoms due to, or following, G. intestinalis infection can significantly impact the quality of life (Painter et al. 2015; Vesy and Peterson 1999; Watkins and Eckmann 2014). Symptom recurrence, including abdominal symptoms and fatigue, can result from reinfection, treatment failure, and disturbances in the gut mucosa or post-infection syndromes (Watkins and Eckmann 2014). In developed countries, these sequelae can have an enormous impact on the quality of life; in developing countries, particularly in children,

they add yet another burden to populations that are already disadvantaged. Infection with G. intestinalis remains latent because only a handful of agents have been used in therapy, and the agents that are available may have adverse effects or be contraindicated in certain clinical situations. Additionally, resistance may play a role in some infections (Vesy and Peterson 1999; Watkins and Eckmann 2014). Thus, research on the development of new compounds to combat giardiasis is needed.

In this work, we demonstrated that synthetic, bLF-derived LFcin17-30, LFampin265-284, and LFchimera have parasiticidal activity against $G$. intestinalis trophozoites in vitro. In 
349 vitro giardicidal activity of native human and bovine LF, as well as their derived $\mathrm{N}$ 350 terminal peptides, has been observed previously (Turchany et al. 1995). Treated 351 trophozoites showed ultrastructural damage, with lactoferrin and its N-terminal peptides 352 causing striking and complex morphologic changes in the trophozoite plasmalemma, 353 endomembrane and cytoskeleton and increasing the electron density of the lysosome-like 354 peripheral vacuoles (Turchany et al. 1997). The synthetic peptides used in this work are 355 different from those reported by Turchany et al. (1995), but the giardicidal activity, the 356 binding and endocytosis of the peptides by the trophozoites and the damage induced at the 357 ultrastructural level were similar. Neither $\mathrm{Fe}^{3+}, \mathrm{Fe}^{2+}$, nor other compounds such as $\mathrm{MgCl}_{2}$, 358 or $\mathrm{CaCl}_{2}$, diminished or prevented the parasiticidal activity of LFpeptides against $G$. 359 intestinalis trophozoites (unpublished results). While we cannot directly compare our 360 results with Turchany et al. $(1995,1997)$ because we used cultures from cysts of $G$. 361 intestinalis directly isolated from patients that were forced to excyst in the laboratory, their 362 results support our conclusion that LFpeptides exhibit potent in vitro antigiardial activity.

LFchimera presented greater giardicidal activity in the viability assays and induced more pronounced damage to $G$. lamblia trophozoites at the ultrastructural level compared to its 366 individual constituent peptides LFcin17-30 and LFampin265-284. All of the peptides at 367 low concentrations had a dramatic parasiticidal effect when they were combined with the 368 pharmacological drugs metronidazole or albendazole (used to treat giardiasis).

370 Regarding differences found with the two methods to estimate viability (PI and CFDA) in 371 G. intestinalis treated with LFpeptides, LF and drugs (Figures 1 A and 1 B, respectively), it 372 is known that the fluorogenic dye PI is unable to traverse intact cell membranes; therefore, 
373 only cells with disrupted or broken membranes are counterstained by PI. Consequently, PI

374 is an indirect indicator of cellular viability. On the other hand, it has been established that

375 an intact lipid bilayer slows the leakage of the fluorochrome CFDA from within intact cells,

376 while injured or stressed cells cannot retain or accumulate the fluorochrome CFDA (dos

377 Santos et al. 2015; Schupp et al. 1988). Additionally, the replication time is different in the

378 distinct genotypes of G. intestinalis; consequently, the drug sensitivity data from in vitro

379 studies in Giardia varies as a function of the replication time and the methodological

380 techniques employed (Hahn et al. 2013). Therefore, both factors (different replication times

381 and methodologies) could explain the differences of results obtained using PI and CFDA

382 (Figures $1 \mathrm{~A}$ and 1B). Despite the differences in these methodologies, it is clear that LF and

383 LFpeptides have a parasiticidal effect on $G$. intestinales isolates, and data obtained by 384 electron microscopy (Figures 3 and 4, respectively) corroborate our conclusion that LF and 385 LF peptides exhibited giardicidal activity.

387 Regarding the mechanism of action, our confocal microscopy observations demonstrated 388 that LFpeptides were bound and internalized by G. intestinalis trophozoites (Figure 2). We 389 speculate that the localization of peptides inside $G$. intestinalis is an event involved in the 390 mechanism of action of LF peptides against this protozoan. It is likely that LFpeptides 391 caused damage to membranes, including internal membranes. In agreement with this idea, 392 LFpeptides appeared to trigger an early programmed cell death or apoptosis-like event in $G$. 393 intestinalis (Figure 5).

395 In higher eukaryotes, programmed cell-death (PCD) is the death of a cell in any form 396 mediated by an intracellular program. Programmed cell death is a genetically regulated 
397

398

399

400

401

402

403

404

405

406

407

408

409

410

411

412

413

414

415

416 417 C-H, Figure 4, panels B-D).

process that is central to the development, homeostasis and integrity of multicellular organisms (Ameisen 2002). Interestingly, several molecules or pathways that regulate PCD in higher eukaryotes have been implicated in the death of unicellular organisms (Bruchhaus et al. 2007), and apoptosis-like programmed cell death (PCD) has been described in multiple primitive eukaryotes and protists, including unicellular parasites, meaning that unicellular organisms can commit suicide in response to various stimuli (Bruchhaus et al. 2007; Kaczanowski et al. 2011; van Zandbergen et al. 2010). G. intestinalis is a divergent amitochondrial eukaryote with a unicellular, bi-nucleated flagellated structure, but even this organism undergoes PCD (Bagchi et al. 2012). However, this is a pathway of autophagy and differs from the classical apoptosis of higher eukaryotes. Annexin-V and 7-AAD staining was used to analyze early programmed cell death in G. intestinalis (Bagchi et al. 2012; Ghosh et al. 2009). Annexin V (or Annexin A5) is a member of the annexin family of intracellular proteins and binds to phosphatidylserine (PS). Externalization of PS is an indicator of early apoptosis-like or programmed cell death in Giardia (Ghosh et al. 2009). 7-AAD (7-amino-actinomycin D) has a high DNA binding constant and is efficiently excluded by intact cells and bound by cells in necrosis or late programmed cell death. Both fluorescent dyes were measured in G. intestinalis using Acoustic Focusing Cytometer AttuneTM Blue/Red (Life Technologies). Our results demonstrate that all of the peptides induced early programmed cell death in G. intestinalis (Figure 5). These data are corroborated by the type of damage observed at the ultrastructural level (Figure 3, Panels

419 There is no previous evidence of apoptosis-like or programmed cell death induced by bLF 420 in a parasite, but it has been reported that LF triggered programmed cell death in cells 
421 infected with influenza virus (Pietrantoni et al. 2010), echovirus (Tinari et al. 2005), and

422 Listeria monocytogenes (Valenti et al. 1999). Additionally, LF triggers apoptosis or 423 apoptosis-like activity in microorganisms such as Saccharomyces cerevisiae (Acosta424 Zaldivar et al. 2016) and Candida albicans (Andres et al. 2008).

425

426 Further studies are needed to determine if LF and LFpeptides have an effect against $G$.

427 intestinalis in in vivo models. However, all data to date suggest that LFpeptides are active 428 compounds with the potential to combat giardiasis, alone or when combined with 429 chemotherapeutic drugs.

\section{Acknowledgments}

432 The authors thank Dr. Martha Ponce-Macotela from LPE-Instituto Nacional de Pediatría for 433 her generous donation of clinical isolates of Giardia intestinalis, Dr. Luis Felipe Jimenez 434 from Laboratorio MET-Facultad de Ciencias UNAM for his efficient technical support, and 435 Jorge A. Canizalez-León for his support in the preparation of images. This work was 436 supported by CONACyT México grant CB-2014-236546 (NLS). Aguilar-Diaz was a PhD 437 student from the Programa Regional del Noroeste para el Doctorado en Biotecnología de la 438 Universidad Autónoma de Sinaloa, and was supported by a PhD fellowship CONACyT439 México (Grant 169048). Kamran Nazmi and Dr. Jan Bolscher were supported through a 440 grant from the University of Amsterdam for research into the focal point Oral Infections 441 and Inflammation. 


\section{References}

446

447

448

449

450

451

452

453

454

455

456

457

458

459

460

461

462

463

464

465

466

467

468

469

470

471

472

473

474

475

476

477

478

479

480

481

482

483

484

485

486

487

488

489
Abdel-Hafeez, E.H., Belal, U.S., Abdellatif, M.Z., Naoi, K., and Norose, K. 2013. Breast-feeding protects infantile diarrhea caused by intestinal protozoan infections. The Korean journal of parasitology 51(5): 519-524. doi: 10.3347/kjp.2013.51.5.519.

Acosta-Zaldivar, M., Andres, M.T., Rego, A., Pereira, C.S., Fierro, J.F., and Corte-Real, M. 2016. Human lactoferrin triggers a mitochondrial- and caspase-dependent regulated cell death in Saccharomyces cerevisiae. Apoptosis : an international journal on programmed cell death $\mathbf{2 1}(2)$ : 163-173. doi: 10.1007/s10495-015-1199-9.

Adam, R.D. 2001. Biology of Giardia lamblia. Clinical microbiology reviews 14(3): 447-475. doi: 10.1128/CMR.14.3.447-475.2001.

Ameisen, J.C. 2002. On the origin, evolution, and nature of programmed cell death: a timeline of four billion years. Cell death and differentiation 9(4): 367-393. doi: 10.1038/sj/cdd/4400950.

Andres, M.T., Viejo-Diaz, M., and Fierro, J.F. 2008. Human lactoferrin induces apoptosis-like cell death in Candida albicans: critical role of K+-channel-mediated K+ efflux. Antimicrobial agents and chemotherapy 52(11): 4081-4088. doi: 10.1128/AAC.01597-07.

Bagchi, S., Oniku, A.E., Topping, K., Mamhoud, Z.N., and Paget, T.A. 2012. Programmed cell death in Giardia. Parasitology 139(7): 894-903. doi: 10.1017/S003118201200011X.

Bolscher, J., Nazmi, K., van Marle, J., van 't Hof, W., and Veerman, E. 2012. Chimerization of lactoferricin and lactoferrampin peptides strongly potentiates the killing activity against Candida albicans. Biochem Cell Biol 90(3): 378-388. doi: 10.1139/o11-085.

Bolscher, J.G., Adao, R., Nazmi, K., van den Keybus, P.A., van 't Hof, W., Nieuw Amerongen, A.V., Bastos, M., and Veerman, E.C. 2009. Bactericidal activity of LFchimera is stronger and less sensitive to ionic strength than its constituent lactoferricin and lactoferrampin peptides. Biochimie 91(1): 123-132. doi: 10.1016/j.biochi.2008.05.019.

Bruchhaus, I., Roeder, T., Rennenberg, A., and Heussler, V.T. 2007. Protozoan parasites: programmed cell death as a mechanism of parasitism. Trends in parasitology 23(8): 376-383. doi: 10.1016/j.pt.2007.06.004.

Carranza, P.G., and Lujan, H.D. 2010. New insights regarding the biology of Giardia lamblia. Microbes and infection / Institut Pasteur 12(1): 71-80. doi: 10.1016/j.micinf.2009.09.008.

Dominguez-Lopez, M.E., Gonzalez-molero, I., Ramirez-Plaza, C.P., Soriguer, F., and Olveira, G. 2011. [Chonic diarrhea and malabsorption due to common variable immunodeficiency, gastrectomy and giardiasis infection: a difficult nutritional management]. Nutricion hospitalaria 26(4): 922-925. doi: 10.1590/S0212-16112011000400037.

dos Santos, S.R., Branco, N., Franco, R.M., Paterniani, J.E., Katsumata, M., Barlow, P.W., and Gallep Cde, M. 2015. Fluorescence decay of dyed protozoa: differences between stressed and nonstressed cysts. Luminescence : the journal of biological and chemical luminescence 30(7): 11391147. doi: 10.1002/bio.2872.

Eckmann, L. 2003. Mucosal defences against Giardia. Parasite immunology 25(5): 259-270.

Elsafi, S.H., Al-Maqati, T.N., Hussein, M.I., Adam, A.A., Hassan, M.M., and Al Zahrani, E.M. 2013. Comparison of microscopy, rapid immunoassay, and molecular techniques for the detection of Giardia lamblia and Cryptosporidium parvum. Parasitology research 112(4): 1641-1646. doi: 10.1007/s00436-013-3319-1.

Escobedo, A.A., Almirall, P., Robertson, L.J., Franco, R.M., Hanevik, K., Morch, K., and Cimerman, S. 2010. Giardiasis: the ever-present threat of a neglected disease. Infectious disorders drug targets 10(5): 329-348. 
Furness, B.W., Beach, M.J., and Roberts, J.M. 2000. Giardiasis surveillance--United States, 1992-

491

492

493

494

495

496

497

498

499

500

501

502

503

504

505

506

507

508

509

510

511

512

513

514

515

516

517

518

519

520

521

522

523

524

525

526

527

528

529

530

531

532

533

534

535

536
1997. MMWR. CDC surveillance summaries : Morbidity and mortality weekly report. CDC surveillance summaries / Centers for Disease Control 49(7): 1-13.

Gendrel, D., Richard-Lenoble, D., Kombila, M., Gendrel, C., and Baziomo, J.M. 1989. Giardiasis and breast-feeding in urban Africa. The Pediatric infectious disease journal 8(1): 58-59.

Ghosh, E., Ghosh, A., Ghosh, A.N., Nozaki, T., and Ganguly, S. 2009. Oxidative stress-induced cell cycle blockage and a protease-independent programmed cell death in microaerophilic Giardia lamblia. Drug design, development and therapy 3: 103-110.

Gillin, F.D., Reiner, D.S., and Wang, C.S. 1983. Killing of Giardia lamblia trophozoites by normal human milk. Journal of cellular biochemistry 23(1-4): 47-56. doi: 10.1002/jcb.240230106.

Hahn, J., Seeber, F., Kolodziej, H., Ignatius, R., Laue, M., Aebischer, T., and Klotz, C. 2013. High sensitivity of Giardia duodenalis to tetrahydrolipstatin (orlistat) in vitro. PloS one 8(8): e71597. doi: 10.1371/journal.pone.0071597.

Hautus, M.A., Kortbeek, L.M., Vetter, J.C., and Laarman, J.J. 1988. In vitro excystation and subsequent axenic growth of Giardia lamblia. Transactions of the Royal Society of Tropical Medicine and Hygiene 82(6): 858-861.

Hill, D.R. 1993. Giardiasis. Issues in diagnosis and management. Infectious disease clinics of North America 7(3): 503-525.

Kaczanowski, S., Sajid, M., and Reece, S.E. 2011. Evolution of apoptosis-like programmed cell death in unicellular protozoan parasites. Parasites \& vectors 4: 44. doi: 10.1186/1756-3305-4-44.

Morrow, A.L., Reves, R.R., West, M.S., Guerrero, M.L., Ruiz-Palacios, G.M., and Pickering, L.K. 1992. Protection against infection with Giardia lamblia by breast-feeding in a cohort of Mexican infants. The Journal of pediatrics 121(3): 363-370.

Nabarro, L.E., Lever, R.A., Armstrong, M., and Chiodini, P.L. 2015. Increased incidence of nitroimidazole-refractory giardiasis at the Hospital for Tropical Diseases, London: 2008-2013. Clinical microbiology and infection: the official publication of the European Society of Clinical Microbiology and Infectious Diseases 21(8): 791-796. doi: 10.1016/j.cmi.2015.04.019.

Ochoa, T.J., Chea-Woo, E., Campos, M., Pecho, I., Prada, A., McMahon, R.J., and Cleary, T.G. 2008. Impact of lactoferrin supplementation on growth and prevalence of Giardia colonization in children. Clinical infectious diseases : an official publication of the Infectious Diseases Society of America 46(12): 1881-1883. doi: 10.1086/588476.

Painter, J.E., Gargano, J.W., Collier, S.A., and Yoder, J.S. 2015. Giardiasis surveillance -- United States, 2011-2012. MMWR supplements 64(3): 15-25.

Perez-Rangel, A., Hernandez, J.M., Castillo-Romero, A., Yepez-Mulia, L., Castillo, R., HernandezLuis, F., Nogueda-Torres, B., Luna-Arias, J.P., Radilla, G., and Leon-Avila, G. 2013. Albendazole and its derivative JVG9 induce encystation on Giardia intestinalis trophozoites. Parasitology research 112(9): 3251-3257. doi: 10.1007/s00436-013-3521-1.

Pietrantoni, A., Dofrelli, E., Tinari, A., Ammendolia, M.G., Puzelli, S., Fabiani, C., Donatelli, I., and Superti, F. 2010. Bovine lactoferrin inhibits influenza A virus induced programmed cell death in vitro. Biometals: an international journal on the role of metal ions in biology, biochemistry, and medicine 23(3): 465-475. doi: 10.1007/s10534-010-9323-3.

Roxstrom-Lindquist, K., Palm, D., Reiner, D., Ringqvist, E., and Svard, S.G. 2006. Giardia immunity-an update. Trends in parasitology 22(1): 26-31. doi: 10.1016/j.pt.2005.11.005.

Schupp, D.G., Januschka, M.M., Sherlock, L.A., Stibbs, H.H., Meyer, E.A., Bemrick, W.J., and Erlandsen, S.L. 1988. Production of viable Giardia cysts in vitro: determination by fluorogenic dye staining, excystation, and animal infectivity in the mouse and Mongolian gerbil. Gastroenterology 95(1): 1-10. 
Stojecki, K., Sroka, J., Karamon, J., Kusyk, P., and Cencek, T. 2014. Influence of selected stool concentration techniques on the effectiveness of PCR examination in Giardia intestinalis diagnostics. Polish journal of veterinary sciences 17(1): 19-25.

Tinari, A., Pietrantoni, A., Ammendolia, M.G., Valenti, P., and Superti, F. 2005. Inhibitory activity of bovine lactoferrin against echovirus induced programmed cell death in vitro. Int J Antimicrob Agents 25(5): 433-438. doi: S0924-8579(05)00060-9 [pii] 10.1016/j.ijantimicag.2005.02.011.

Turchany, J.M., Aley, S.B., and Gillin, F.D. 1995. Giardicidal activity of lactoferrin and N-terminal peptides. Infection and immunity 63(11): 4550-4552.

Turchany, J.M., McCaffery, J.M., Aley, S.B., and Gillin, F.D. 1997. Ultrastructural effects of lactoferrin binding on Giardia lamblia trophozoites. J Eukaryot Microbiol 44(1): 68-72.

Valenti, P., Greco, R., Pitari, G., Rossi, P., Ajello, M., Melino, G., and Antonini, G. 1999. Apoptosis of Caco-2 intestinal cells invaded by Listeria monocytogenes: protective effect of lactoferrin. Experimental cell research 250(1): 197-202. doi: 10.1006/excr.1999.4500.

van Zandbergen, G., Luder, C.G., Heussler, V., and Duszenko, M. 2010. Programmed cell death in unicellular parasites: a prerequisite for sustained infection? Trends in parasitology 26(10): 477483. doi: 10.1016/j.pt.2010.06.008.

Vázquez-Nin, G.H., and Echeverría, O.M. 2000. Introducción a la Microscopía Electrónica Aplicada a las Ciencias Biológicas. Universidad Nacional Autónoma de México Fondo de Cultura Económica. México City.

Vesy, C.J., and Peterson, W.L. 1999. Review article: the management of Giardiasis. Alimentary pharmacology \& therapeutics 13(7): 843-850.

Watkins, R.R., and Eckmann, L. 2014. Treatment of giardiasis: current status and future directions. Current infectious disease reports 16(2): 396. doi: 10.1007/s11908-014-0396-y.

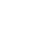


572 573

574 575 576

\section{Figure legends}

Figure 1. LFcin17-30, LFampin265-284 and LFchimera have parasiticidal activity against Giardia intestinalis trophozoites. G. intestinalis cultures were incubated with $100 \mu \mathrm{M}$ Metronidazole or $5 \mu \mathrm{M}$ Albendazole (used as negative controls of viability) or $40 \mu \mathrm{M}$ LFpeptides for $2 \mathrm{~h}$ at $37^{\circ} \mathrm{C}$ (Panel A), or with different concentrations of Metronidazole (used as a negative control of culture growth), LF, or LFpeptides for $12 \mathrm{~h}$ at $37^{\circ} \mathrm{C}$ (Panel B). Then, the samples were washed and viability or culture growth was determined by the exclusion of the dye PI (A) or inclusion of CFDA (B). In both cases (A and B), untreated cultures were used as positive controls of viability or growth. Experiments were performed at least three times in triplicate. The means and standard deviations are indicated in percentages. A value of $\mathrm{P}<0.05$ was considered statistically significant $(*)$.

Figure 2. LFcin17-30, LFampin265-284 and LFquimera are internalized by Giardia intestinalis. Trophozoites were untreated (A) or treated with $2 \mu \mathrm{M}$ FITC-LFpeptides for 30 min at $37^{\circ} \mathrm{C}$ : LFcin17-30 (B), LFampin265-284 (C), and LFchimera (D). Then, samples were fixed, washed and permeabilized with $0.5 \%$ triton X-100 and counterstained with PI (red color). Finally, samples were processed for analysis by confocal microscopy. Arrows show the green fluorescence due to the FITC-LFpeptides (B-D). Bars 20 and $50 \mu \mathrm{m}$.

Figure 3. LFcin17-30, LFampin265-284 and LFchimera cause damage to Giardia intestinalis. Trophozoites were untreated (A, B) or treated with $40 \mu \mathrm{M}$ LFcin17-30 (C, D), LFampin265-284 (E, F), or LFchimera $(\mathrm{G}, \mathrm{H})$, for $2 \mathrm{~h}$ at $37{ }^{\circ} \mathrm{C}$. Then, samples were 
595 processed for analysis by transmission electron microscopy. The sections were analyzed 596 and photographed using a JEOL 1010 transmission electron microscope at $80 \mathrm{kV}$.

597 Untreated cells had a smooth cellular membrane (cm, double-headed arrow), peripheral 598 vacuoles (pv, lines) near the cellular membrane, three pairs of flagella ( $\mathrm{F}$, discontinuous 599 arrow), adherent disk (ad), and two nuclei (N) and two nucleoli (no) (Figure 3, panel A). 600 Magnification of the picture shows granules of electron-dense material (asterisk) distributed 601 in the cytoplasm and the arrangement of the microtubules belonging to the adherent disk 602 (ad) (panel B). Exposure to LFcin17-30 led to profound intracellular changes, such as an 603 increase in electron-dense material in the cytoplasm (asterisk), reorganization of the flagella 604 (F, discontinuous arrow) and displacement of the adherent disk (ad, arrow), Figure 3, panel 605 C. In the magnified picture, there are no peripheral vacuoles (pv) near the cellular 606 membrane (cm, double-headed arrow), Panel D. Treatment with LFampin265-284 also 607 caused intracellular damage, including an increase in electron-dense material (asterisk), 608 reorganization of the flagella ( $\mathrm{F}$, discontinuous arrow), a large hole in the cytoplasm 609 (arrowhead), and also disruption in the cellular membrane (cm, double-headed arrow) 610 (Figure 3, panel E). The magnified picture shows the large hole induced by treatment with 611 LFampin265-284 (arrowhead, panel F). Trophozoites treated with LFchimera produced the 612 most significant changes and damage in the trophozoites. There were marked changes in 613 the electron-dense material in the cytoplasm (asterisks), the flagella (F, discontinuous 614 arrow) were disrupted and reorganized, and the cytoplasm showed large holes (arrowheads) 615 in which some electron-dense material is visible (Figure 3, panel G). In the magnified 616 picture the larges holes (arrowheads) with aggregates inside them (asterisks) can be seen in 617 more detail (Panel H). Shrunken and distorted peripheral vacuoles (pv, lines) were also 618 observed. 
619 Figure 4. LFcin17-30, LFampin265-284 and LFchimera cause damage to Giardia 620 intestinalis. Trophozoites were untreated (A) or treated with $40 \mu \mathrm{M}$ of LFcin17-30 (B), 621 LFampin265-284 (C), or LFchimera (D) for $2 \mathrm{~h}$ at $37^{\circ} \mathrm{C}$. Then, samples were processed for 622 analysis. Specimens were observed under a scanning electron microscope JEOL 623 LSM6360LV. Trophozoites treated with LF peptides show alterations in size, irregular 624 form and perforations (arrows, B-C), compared with untreated trophozoites (which had the 625 typical structure of $G$. intestinalis trophozoites, A). Trophozoites treated with LFchimera 626 exhibited aggregates or vesicles and several had a large hole in their membranes (arrow, 627 panel D).

628

629 Figure 5. Programmed cell death in Giardia intestinalis induced by LFpeptides. 630 Trophozoites of $G$. intestinalis were treated with $40 \mu \mathrm{M}$ of LFcin17-30 (A), LFampin265631284 (B) or LFchimera (C) for $2 \mathrm{~h}$ at $37^{\circ} \mathrm{C}$. Samples were stained with Allophycocyanin632 Annexin (Annexin-APC, to stain Annexin V) and 7-Amino-Actinomicyn D (7-AAD), 633 processed and analyzed by flow cytometry. Q1: Necrotic cells, Q2: Necroptotic cells, Q3: 634 Earlier apoptotic cells. Q4: live cells. One of three representative experiments is shown. 635 636 637 638 639 640 641 642 
645

646

\begin{tabular}{|c|c|c|c|c|}
\hline Compounds & $\begin{array}{c}\text { Concentrations } \\
(\boldsymbol{\mu M})\end{array}$ & $\begin{array}{c}\text { LFcin } \\
\mathbf{1 7 - 3 0} \\
(\boldsymbol{\mu M})\end{array}$ & $\begin{array}{c}\text { LFampin } \\
\mathbf{2 6 5 - 2 8 4} \\
(\boldsymbol{\mu M})\end{array}$ & $\begin{array}{c}\text { LFchimera } \\
(\boldsymbol{\mu M})\end{array}$ \\
\hline Metronidazole & $\mathbf{1 0 0}$ & - & - & - \\
Metronidazole & 20 & 20 & - & - \\
Metronidazole & 20 & - & 30 & - \\
Metronidazole & 20 & - & - & 5 \\
Albendazole & $\mathbf{5}$ & - & - & - \\
Albendazole & 3 & 20 & & \\
Albendazole & 3 & & 30 & \\
Albendazole & 3 & & & - \\
LFcin17-30 & $\mathbf{8 0}$ & - & - & - \\
LFampin265-284 & $\mathbf{8 0}$ & - & - & - \\
LFchimera & $\mathbf{4 0}$ & - & - & - \\
\hline
\end{tabular}

647

648

649

650

651

652

653

654

655

656

657

658

659

660 
Figure 1

A
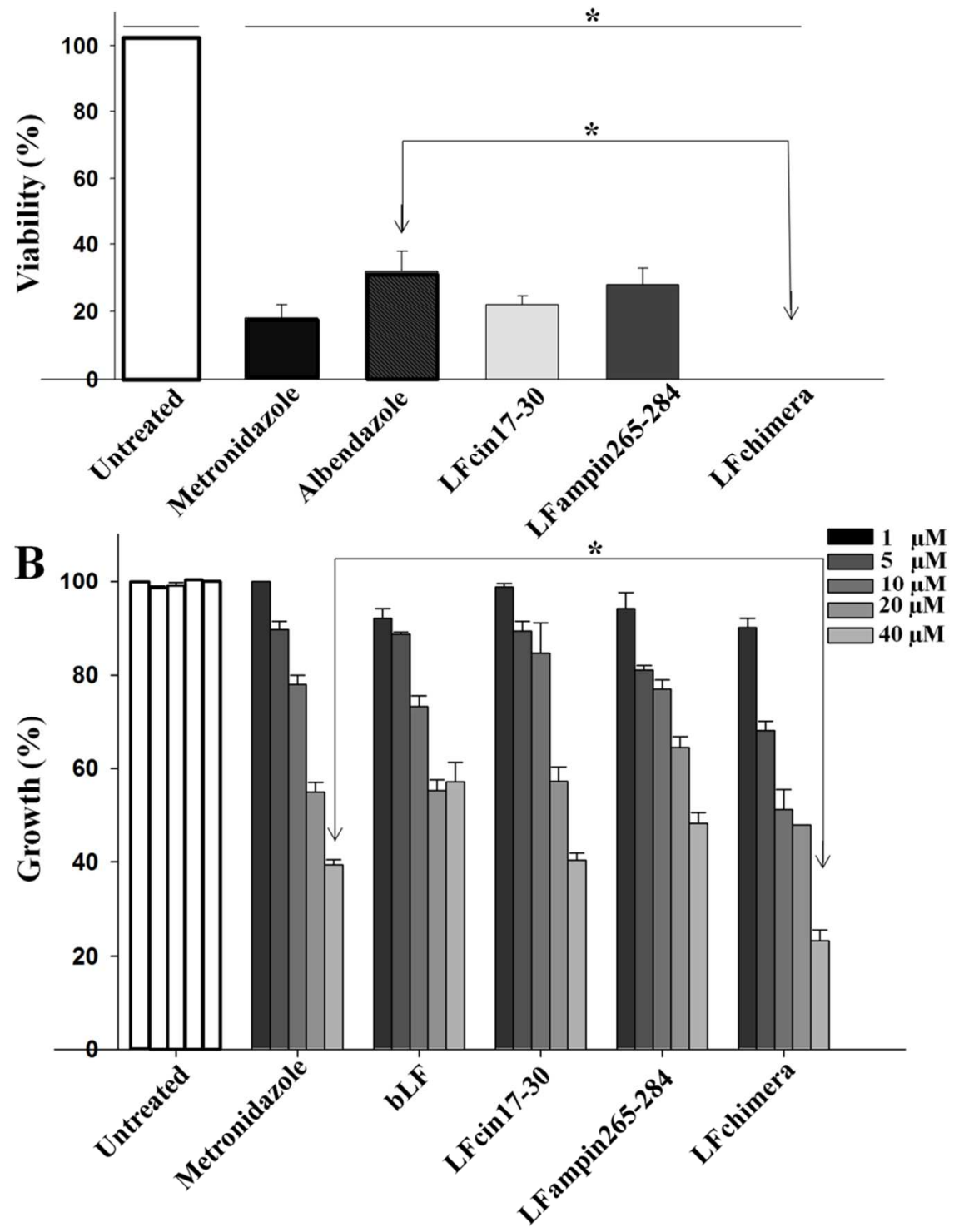

662

663

664

665

666 
$670 \quad$ Figure 2

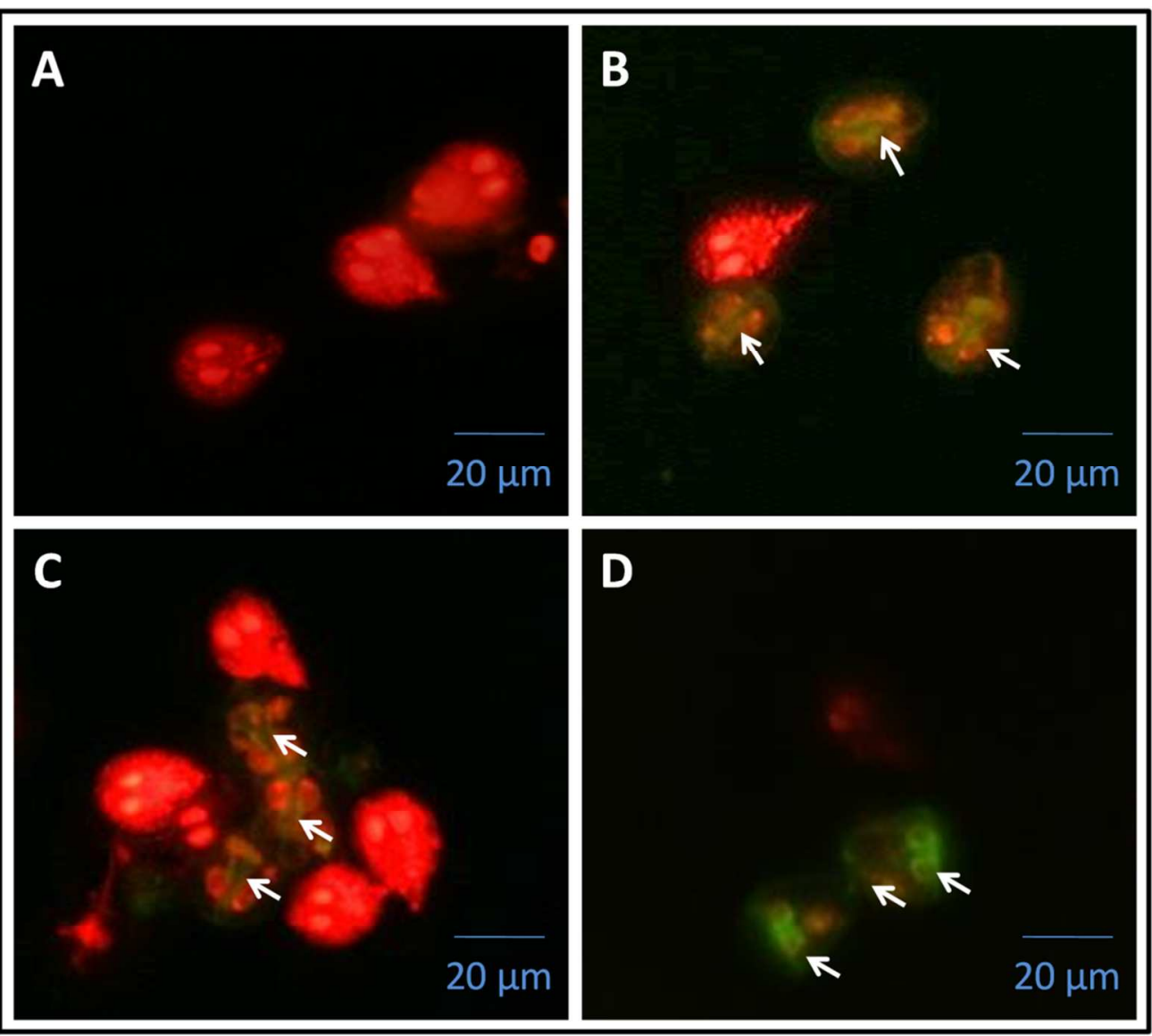

671

672

673

674

675

676

677

678

679

680

681 
$683 \quad$ Figure 3
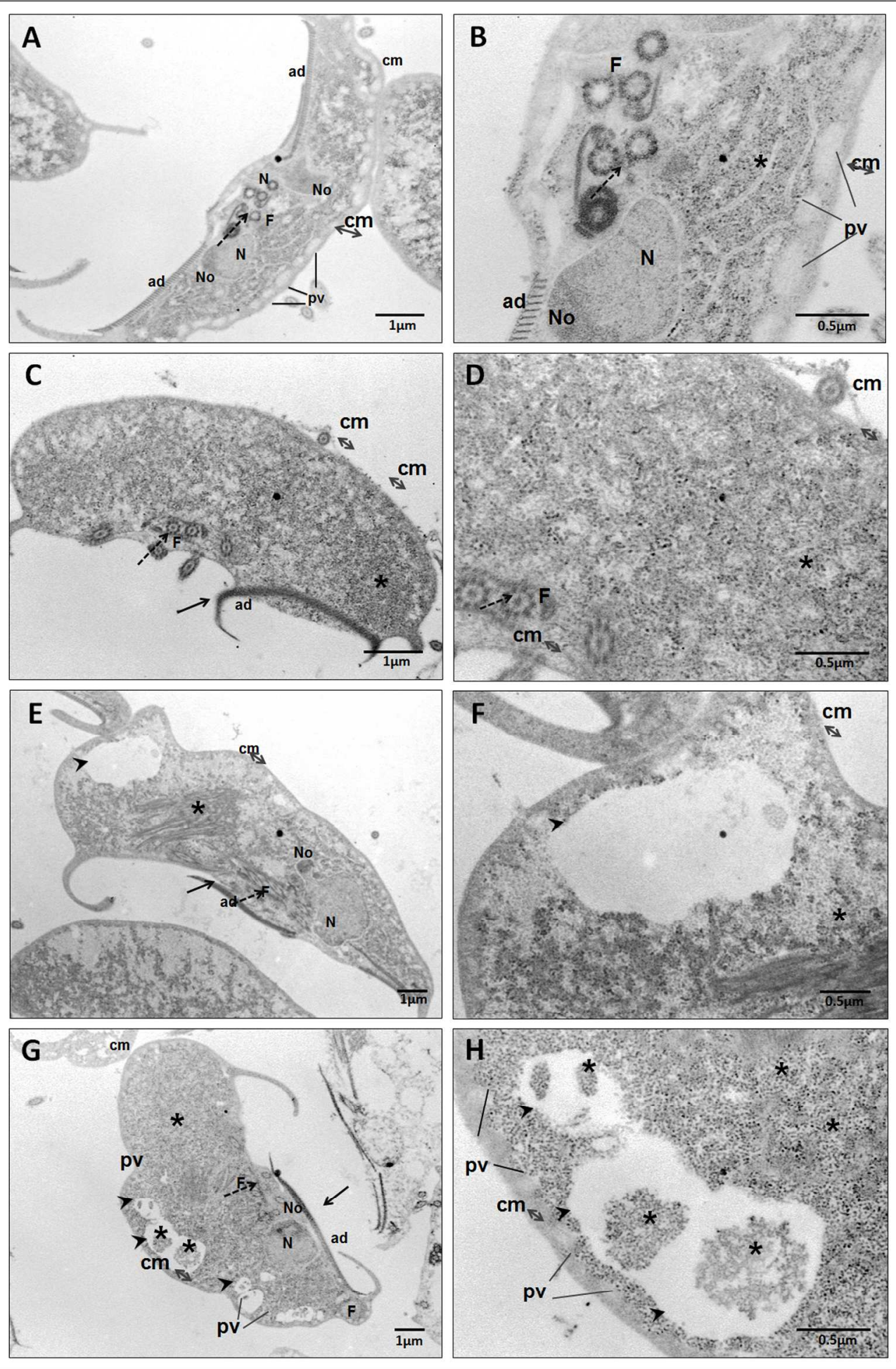
686

687

$688 \quad$ Figure 4

689

690

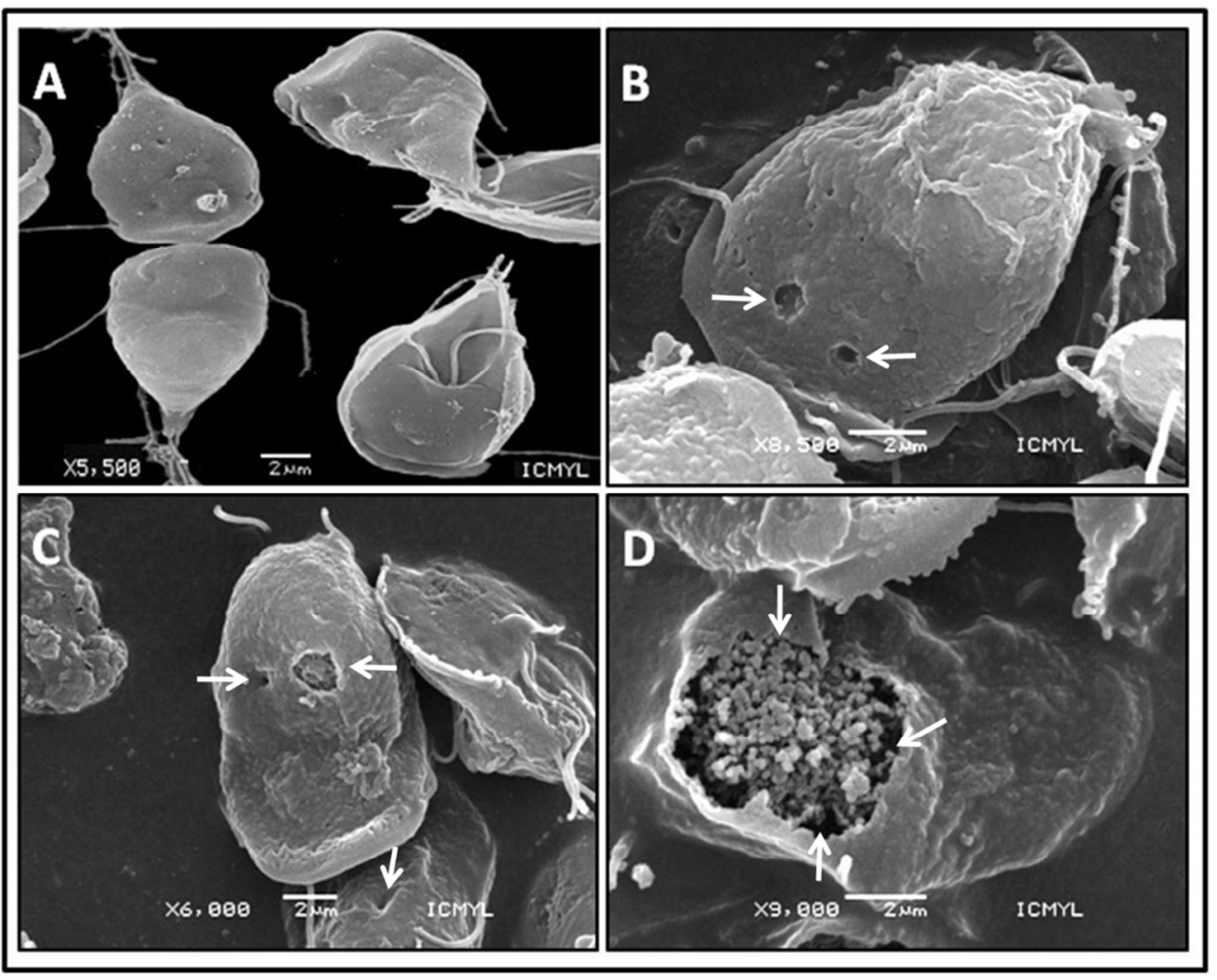

691

692

693

694

695

696

697

698

699 
$701 \quad$ Figure 5
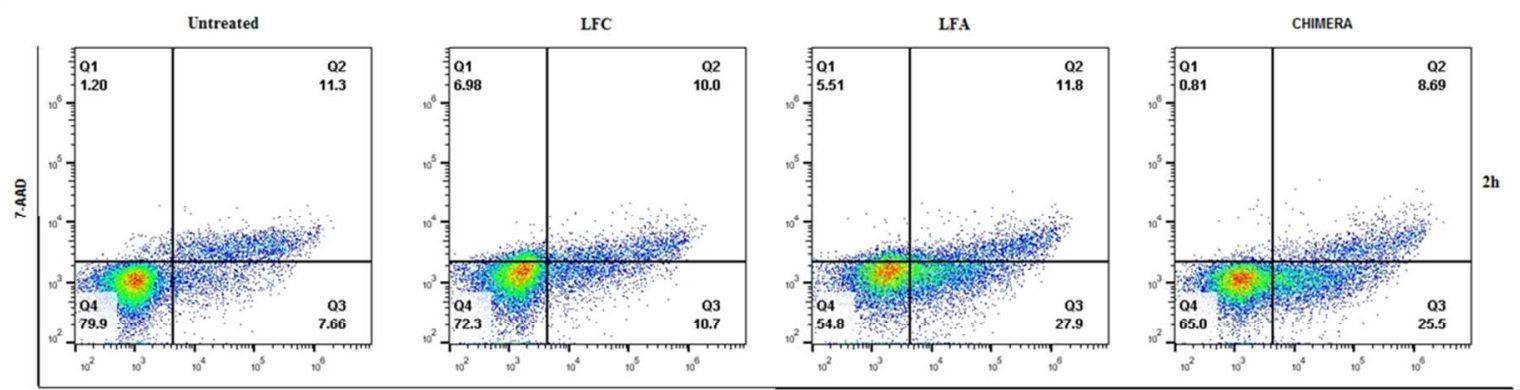

702

Annexin V.APC 